\title{
Polyphenol-Functionalized Plant Viral-Derived Nanoparticles Exhibit Strong Antimicrobial and Antibiofilm Formation Activities
}

\author{
Edith Velázquez-Lam, Juan Imperial, and Fernando Ponz*
}

Cite This: ACS Appl. Bio Mater. 2020, 3, 2040-2047

Read Online

ABSTRACT: (-)-Epigallocatechin gallate (EGCG) is a polyphenolic compound that shows a number of health-promoting effects, especially a broad antimicrobial activity. Virus-derived nanoparticles (VNPs) represent a promising drug carrier since they possess properties like biodegradability and their surface and interior are highly modifiable. Turnip mosaic virus (TuMV) VNPs offer an attractive number of conjugation sites on the external surface. EGCG-TuMV VNPs were synthesized by Mannich condensation, and their antimicrobial activities against the model bacteria Sarcina lutea, Pseudomonas aeruginosa, and Dickeya dadantii were tested. EGCG-TuMV VNPs did not only maintain TuMV structure but also showed an enhanced antimicrobial activity over that found with free EGCG for all of the bacteria

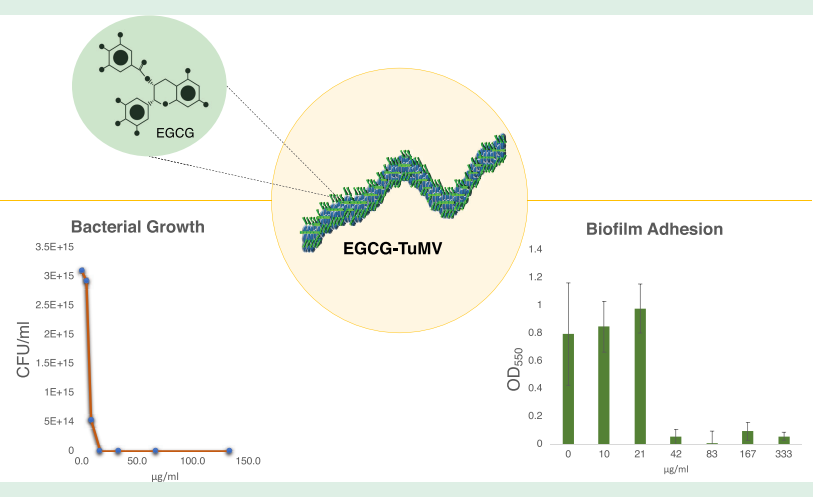
tested. Biofilm formation by P. aeruginosa was also inhibited by EGCG-TuMV VNPs, contrary to free EGCG, which induced higher amounts of biofilm mass in a concentration-dependent manner. Taken together, our results open highly promising perspectives for the antimicrobial exploitation of EGCG-TuMV VNPs.

KEYWORDS: viral nanoparticles, epigallocatechin gallate, polyphenols, tannins, antimicrobial activity, virus-derived particles

\section{INTRODUCTION}

Microbial control is a growing global issue affecting human, animal, and environmental health. There are different aspects of this crisis that make it harder to overcome, such as the existence of multiple antimicrobial resistance mechanisms for survival, or the changing regulations for antimicrobial agents due to increasing environmental awareness. All these together cause a reduction in the effectiveness of current approaches for attacking infections, which leads to economic, environmental, and life losses. This reality marks the increasing need for novel, potent agents that can replace or boost the activity of the existing antimicrobials. ${ }^{1}$

Antimicrobial resistance happens naturally in bacteria and other microbes. It is a dynamic threat since bacteria are capable of evolving, acquiring, and expressing resistance genes, which, ultimately, leads to the selection of resistant microbial clones. ${ }^{2}$ Although antimicrobial resistance happens naturally, there are different reasons that accelerate its development. The misuse of antimicrobial drugs over time has led to the emergence and spread of multidrug-resistant bacteria. This is compounded by a scarcity of newer drugs attributable to restrictive regulatory requirements. ${ }^{3}$

Biofilm formation is another important area to consider when dealing with antimicrobials and antimicrobial resistance. Bacterial biofilms are ubiquitous, multicellular aggregates, usually attached to biotic or abiotic surfaces, within which bacteria are embedded in an extracellular matrix of selfproduced polymers. ${ }^{4}$ Biofilm bacteria usually display higher resistance to antimicrobials than planktonic bacteria due to low penetration and accumulation of antimicrobials in the biofilm and to conditions within the biofilm environment that inactivate antimicrobials. As a result, bacterial biofilms are refractory to antibiotic treatment and to immune responses; therefore, biofilms of pathogenic bacteria play a crucial role in chronic infections.

Antimicrobial control is also an issue in the agricultural sector. Due to a number of factors such as the increasing food demand, climate change, and regulatory limitations in plant protection products, we are witnessing an emergence of new plant diseases that cause significant crop losses. ${ }^{5}$ Current control measures involve the preventive use of traditional chemicals, copper compounds, or antibiotics that are, nonetheless, losing their efficacy due to bacterial resistance. The environmental effects of these control methods are also to be considered. For instance, the traditional use of copper

Received: December 16, 2019

Accepted: March 10, 2020

Published: March 10, 2020

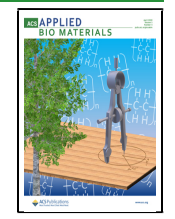


compounds raises concerns, since $95 \%$ of $\mathrm{Cu}$ released into the environment will accumulate in soil and aquatic sediments to potentially toxic levels $(>50-500 \mu \mathrm{g} / \mathrm{mL}){ }^{6}$ These reasons highlight the need for the development of new strategies for plant disease control. These strategies need to be not only effective but also have minimal effects on the environment.

Plant-derived polyphenolic compounds have been reported to have a wide range of biological activities, among which are their antimicrobial and antibiofilm activities against a wide range of microorganisms, including bacterial, fungal, and viral pathogens. ${ }^{7}$ Studies conducted over the last 20 years have shown that the green tea polyphenolic catechins can inhibit the growth of a wide range of Gram-positive and Gram-negative bacterial species with moderate potency. (-)-Epigallocatechin gallate (EGCG) is the most abundant of these, comprising about $50 \%$ of the green tea catechin pool, while (-)-epigallocatechin (EGC) accounts for around 20\%, (-)-epicatechin gallate (ECG) for 13\%, and (-)-epicatechin (EC) for 6\%. ${ }^{8}$ Among these, EGCG is the most bioactive and the most studied. A cup of green tea may contain 100-200 mg EGCG, while catechin and gallocatechin are present in lower amounts.

Since EGCG is sensitive to $\mathrm{pH}$, temperature, and oxygen ${ }^{10,11}$ there are limitations on how this molecule can be used in commercial applications. The design of a vehicle that maintains EGCG's attributed effects is an attractive strategy to overcome its lack of stability.

Incorporation of EGCG into nanoparticles (NPs) has been increasingly reported as a strategy to promote the biological activities of the compound. Inorganic nanoparticles, especially metallic ones (silver, copper, gold, and zinc oxide), have been explored as carriers for antimicrobials. ${ }^{12-15}$ These types of nanoparticles suffer from a number of limitations related to their environmental effects. Absorption of metals may have a negative effect on the structure and function of the photosynthetic apparatus. ${ }^{16}$ Metallic nanoparticles may have an ease for oxidation and aggregation and are difficult to extract and purify after green synthesis. ${ }^{17,18}$ Also, they tend to accumulate in tissues and interact with proteins and DNA, which contributes to the toxicity that affects different organs within the human body. Metallic nanoparticles are also known to produce stimulation of cytokines that contributes to cytotoxicity, immunotoxicity, and genotoxicity. ${ }^{19}$ To prevent chronic toxicity elicited by metallic NPs, organic nanoparticles have been developed as drug delivery carriers. For the above reasons, organic nanoparticles have been favored for biological applications; hence, a number of organic nanoparticles have been tried as carriers for EGCG in different biological systems. These include lipidic, ${ }^{20-22}$ polysaccharide, ${ }^{23,24}$ or proteinaceous nanoparticles. ${ }^{25-27}$ Proteinaceous nanoparticles have received relatively lesser attention, although casein-based nanoparticles have been explored, especially in the nutraceutical area. ${ }^{27,28}$ In one form or another, underlying reasons to try nanoparticle formulations for EGCG relate to the lack of stability of the compound in its free form.

Viral plant-derived nanoparticles (VNPs) are a selfassembly-competent protein structure, the capsid, made out of multiple copies of one or a few types of protein subunits. VNPs have a series of advantages that make them suitable for nanoscale applications. Their proteic nature makes them biodegradable and capsids can be produced in large quantities by infection of plants, hence their production is affordable and scalable. Finally, their surface is highly modifiable to target different molecules. ${ }^{29}$

Specifically, Turnip mosaic virus (TuMV) particles are of interest since their architecture allows for different types of functionalizations. TuMV is a flexuous, filamentous plant virus that belongs to the Potyvirus genus. It is about $700 \mathrm{~nm}$ long, $12 \mathrm{~nm}$ in diameter, and comprises approximately 2000 identical copies of $33 \mathrm{kDa} \mathrm{CP}$ protein subunits that cover a molecule of viral RNA of approximately $10 \mathrm{~kb}$ size. ${ }^{30}$ VNPs can be modified genetically or chemically to expose peptides or small molecules (like catechins) at the surface of the virus. There are 21 lysyl residues present in the CP protein, which are potential targets for chemical functionalization. ${ }^{30}$ This multimeric presentation of EGCGs on the VNPs surface might boost its antimicrobial and antibiofilm activity against several Gram-positive and Gram-negative bacteria.

TuMV-VNPs constitute a versatile tool for the generation of a technological platform with the potential to impact a vast array of areas. Given the pressing need for creating new antimicrobial agents, VNPs are promising, since they have the potential to transport molecules with antimicrobial activity in a more efficient manner. In this study, we show that EGCGs can be efficiently conjugated to VNPs and that these EGCG-VNPs display enhanced antimicrobial activities, as compared to that of the free antimicrobial.

\section{EXPERIMENTAL SECTION}

Production and Purification of TuMV VNPs. Turnip mosaic virus (TuMV, isolate UK 1) was produced and purified as described by Sánchez and Ponz. ${ }^{31}$

Chemical Conjugation of EGCG. EGCG was chemically bound to VNPs through a Mannich reaction. Previously, a buffer exchange was required to eliminate the amines present in TRIS buffer. VNPs were centrifuged $\left(50 \mathrm{~min}, 80000 \times \mathrm{g}, 4{ }^{\circ} \mathrm{C}\right)$ and resuspended in 10 mM $N$-(2-hydroxyethyl)piperazine- $N^{\prime}$-ethanesulfonic acid (HEPES), $\mathrm{pH} 7.5$, to a final concentration of $2 \mathrm{mg} / \mathrm{mL}$. EGCG (Sigma-Aldrich, Germany) was dissolved in 2-morpholinoethanesulfonate (MES), $\mathrm{pH}$ 6.7 , to a final concentration of $5 \mathrm{mg} / \mathrm{mL}$. Different amounts of EGCG were added to $100 \mu \mathrm{g}$ of VNPs and $5 \mu \mathrm{L}$ of $37 \%$ formaldehyde, and the final volume was adjusted to $115 \mu \mathrm{L}$. The reaction was stirred and incubated at room temperature for $72 \mathrm{~h}$. The excess EGCG was removed by centrifugation $\left(50 \mathrm{~min}, 80000 \times g, 4{ }^{\circ} \mathrm{C}\right.$ ), the pellet was washed twice, and resuspended in $10 \mathrm{mM}$ HEPES, $\mathrm{pH} 7.5$, to a final concentration of $1 \mathrm{mg} / \mathrm{mL}$ of VNPs.

EGCG Coupling Estimation. The amount of EGCG bound to TuMV was analyzed by modifying a previously described method for the determination of tannin-protein complexes. ${ }^{32,33}$ To microwells from a 96-well plate, $15 \mu \mathrm{L}$ of portions of EGCG-VNPs solution was added and incubated with $15 \mu \mathrm{L}$ of $10 \mathrm{mM}$ HEPES, pH 7.5, $90 \mu \mathrm{L}$ of SDS-trietanolamine solution (SDS $1 \%(\mathrm{w} / \mathrm{v})$, trietanolamine $7 \%(\mathrm{v} /$ $\mathrm{v})$ ), and $30 \mu \mathrm{L}$ of a ferric chloride reagent (0.01 M ferric chloride in $0.1 \mathrm{M} \mathrm{HCl}$ ) for $30 \mathrm{~min}$, after which absorbance at $510 \mathrm{~nm}$ was recorded. A calibration curve was constructed by plotting absorbances for different, known amounts of EGCG.

Electron Microscopy. Electron microscopy grids (400 mesh nickel, carbon-coated) were prepared by gently placing them, filmdown, on top of the antiserum drop and incubated for $10 \mathrm{~min}$ at room temperature. Grids were then removed with tweezers and rinsed with the same buffer. They were later floated on a $10 \mu \mathrm{L}$ drop of VNPs $(0.05 \mathrm{mg} / \mathrm{mL})$, incubated at room temperature for $15 \mathrm{~min}$, and washed with buffer. Finally, the grids were rinsed with distilled water and stained with $2 \%$ uranyl acetate for $2 \mathrm{~min}$. The samples were examined on a transmission electron microscope (JEM JEOL 1010, Japan).

Assay for Antimicrobial Activity of TuMV-EGCG VNPs. The antimicrobial activity of EGCG-VNPs was evaluated against Dickeya dadantii, Sarcina lutea, and Pseudomonas aeruginosa by disk 
susceptibility tests. Overnight bacterial cultures were grown in Mueller-Hinton media $(\mathrm{MH})$, adjusted to an $\mathrm{OD}_{600}$ of 0.1 , and inoculated on Muller-Hinton agar (MHA) plates. Antibiotic disks containing $10 \mu \mathrm{g}$ of EGCG-VNPs or $5 \mu \mathrm{g}$ of kanamycin (as control) were placed on the plates and incubated for $24 \mathrm{~h}$ at $37^{\circ} \mathrm{C}$.

To evaluate the ability of EGCG-VNPs to inhibit bacterial growth in liquid media, overnight cultures were grown on $\mathrm{MH}$ (for S. lutea), or minimal M9 medium containing $48 \mathrm{mM} \mathrm{Na} 2 \mathrm{HPO}_{4}, 22 \mathrm{mM}$ $\mathrm{KH}_{2} \mathrm{PO}_{4}, 9 \mathrm{mM} \mathrm{NaCl}, 19 \mathrm{mM} \mathrm{NH}{ }_{4} \mathrm{Cl}, \mathrm{pH}$ 7, supplemented with 2 $\mathrm{mM} \mathrm{MgSO}_{4}, 100 \mu \mathrm{M} \mathrm{CaCl}_{2}$, and $20 \mathrm{mM}$ glucose (for D. dadantii and $P$. aeruginosa). The overnight cultures were adjusted to $\mathrm{OD}_{600}$ of 0.1 and $150 \mu \mathrm{L}$ portions were placed in microwells from a 96-well plate. Different amounts of EGCG-VNPs and free EGCG were added. The plates were incubated in a shaker $(200 \mathrm{rpm})$ for $24 \mathrm{~h}$ at $37^{\circ} \mathrm{C}$. After incubation, absorbance was recorded at $600 \mathrm{~nm}$ and a single plateserial dilution spotting (SP-SDS) was performed. ${ }^{34}$ The results of three plates with three replicates corresponding to a particular sample were averaged, and this value was regarded as the minimal inhibitory concentration (MIC) of EGCG-VNPs against each microorganism.

Biofilm Formation Assays. For the evaluation of the inhibition of biofilm formation by $P$. aeruginosa, the $\mathrm{OD}_{600}$ of an overnight culture was adjusted to of 0.1 with minimal M9 medium. One hundred fifty microliter portions were placed in microwells from a 96well plate. The plates were incubated in a shaker $(200 \mathrm{rpm})$ for $24 \mathrm{~h}$ at $37{ }^{\circ} \mathrm{C}$. After incubation, microwells were carefully washed three times to remove planktonic cells. One hundred eighty microliters of crystal violet $2 \%(\mathrm{w} / \mathrm{v})$ in methanol were added and incubated at room temperature for $10 \mathrm{~min}$. Wells were washed three times to remove excess dye, $180 \mu \mathrm{L}$ of ethanol was added, and absorbance was recorded at $550 \mathrm{~nm}$.

\section{RESULTS AND DISCUSSION}

Chemical Conjugation of EGCG. Although a large number of health-promoting benefits have been attributed to EGCG, their transfer toward specific applications has proven limited. The incorporation of EGCG into nanoparticles is a promising strategy to help the bioavailability and, as a result, opens the path for the exploitation of its many beneficial activities. Protein-based nanoparticles, in particular VNPs, could represent a promising alternative, in view of their lack of such environmental disadvantages and of their highly modifiable surface, which allows high flexibility on the design of VNPs. The shape of TuMV makes it an attractive candidate to be used as a nanocarrier. As previously reported, lysine residues from the TuMV capsid protein are mainly exposed on the surface and can be used in the functionalization of the virion. $^{35}$ Through lysine conjugation, EGCG molecules bound to VNPs will be likewise exposed to the outside environment (Figure 1). This EGCG exposure toward the exterior of the nanoparticle is important since it has been suggested that the negatively charged EGCG binds to the positively charged external surface of cell membranes, thus damaging or fragmenting the lipid bilayer. ${ }^{36}$ This configuration would allow for a high number of available EGCG molecules concentrated within a relatively small area (high effective concentration). To obtain such EGCG-TuMV nanoparticles, a Mannich condensation was used to target both the polyphenolic compound and primary amines from lysine residues, as shown in Figure 2.

The ratio of EGCG to VNP in the reaction was optimized by testing different concentrations of EGCG. After reacting, unbound EGCG was removed by ultracentrifugation and the resulting conjugated EGCG was estimated. The TuMV concentration was kept constant at $0.87 \mu \mathrm{g} / \mu \mathrm{L}$. As depicted in Figure 3, an EGCG concentration of $2.27 \mathrm{mM}$ in the reaction yielded a high amount of conjugated EGCG. One

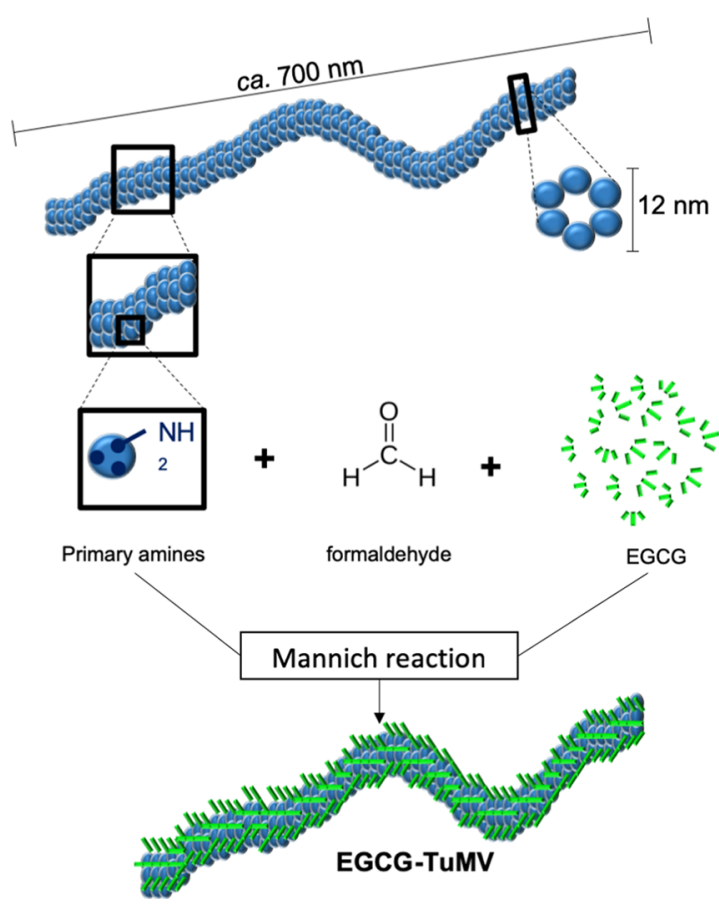

Figure 1. Schematic representation of EGCG-TuMV nanoparticle synthesis. The step of discarding the excess of reagents not incorporated into particles is not represented.

higher initial concentration of initial EGCG in the series was tested $(2.66 \mathrm{mM})$, but this was too close to concentrations resulting in a high degree of protein precipitation (results not shown), a situation to be expected since aggregation from the binding at high EGCG loading capacities has been described. ${ }^{28}$ Thus, we decided to work at $2.27 \mathrm{mM}$. At these chosen working conditions, the estimated amount of EGCG coupled to TuMV was $1.1 \pm 0.1 \mu \mathrm{g}$ EGCG/ $\mu \mathrm{g}$ TuMV. These conjugated VNPs were used in subsequent biological assays.

Although EGCG is known to interact noncovalently with proteins, $^{37}$ as seen in Figure S2, those VNPs that were produced using formaldehyde yielded on a higher amount of linked EGCG. Since polyphenols are known to be highly reactive with proteins and tend to aggregate, ${ }^{38}$ an important consideration was the maintenance of TuMV particle structure. TEM images, shown in Figure 3, did not reveal significant changes in the VNP structure after EGCG conjugation. The characteristic filamentous shape of TuMV particles remained largely unmodified.

Antimicrobial Activity of EGCG-TuMV VNPs. Once EGCG-TuMV conjugation was completed, the maintenance of EGCG-derived antimicrobial activity was tested. First, a disk diffusion susceptibility test was performed on MHA plates. As shown in Figure 4, there was a good antimicrobial activity for both $D$. dadantii and $S$. lutea. In both cases, we found inhibition around disks containing $10 \mu \mathrm{g}$ of EGCG-TuMV.

To establish the limits of the antimicrobial properties, tests in liquid media were performed. It has been previously reported that EGCG shows lower antimicrobial activity against Gram-negative bacteria due to their outer layer of liposaccharides. $^{39}$ D. dadantii and P. aeruginosa (Gram-negative) and S. lutea (Gram-positive) were compared. Experiments were repeated three times to ensure reproducibility with three replicates each. Cultures were performed in 96-well microtiter plates with minimal M9 medium supplemented with glucose 
<smiles>O=C(O)c1cc(O)c(O)c(O)c1</smiles><smiles>Cc1cc(O)c(O)c(O)c1</smiles><smiles>[C+]C=O</smiles>

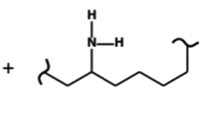

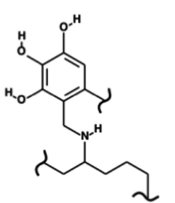

EGCG Lysine or $\mathrm{N}$ - terminal

Figure 2. Mannich condensation between EGCG and TuMV.

(a)

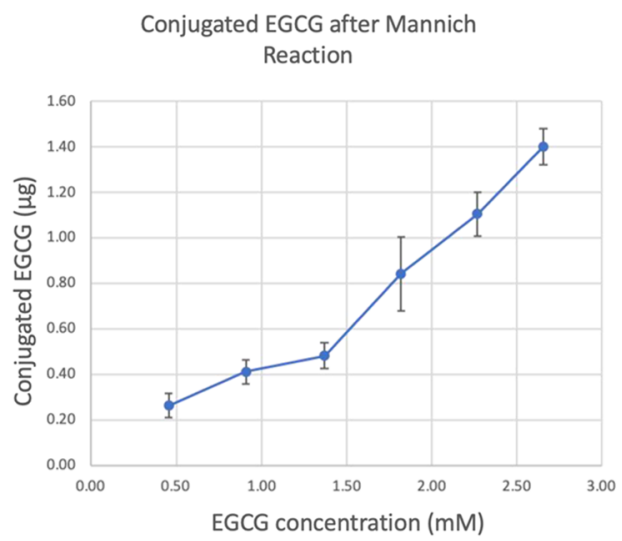

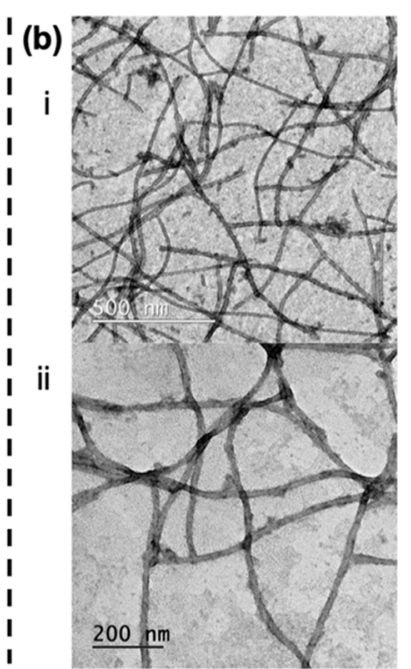

Figure 3. EGCG-TuMV characterization. (a) Amount ( $\mu \mathrm{g})$ of EGCG conjugated to TuMV as a function of EGCG concentration in the initial condensation reaction. TuMV concentration was maintained constant in the reaction at $0.87 \mathrm{mg} / \mathrm{mL}$. (b) Electron microscopy (TEM) micrographs of purified TuMV (i) or EGCG-TuMV particles (ii).

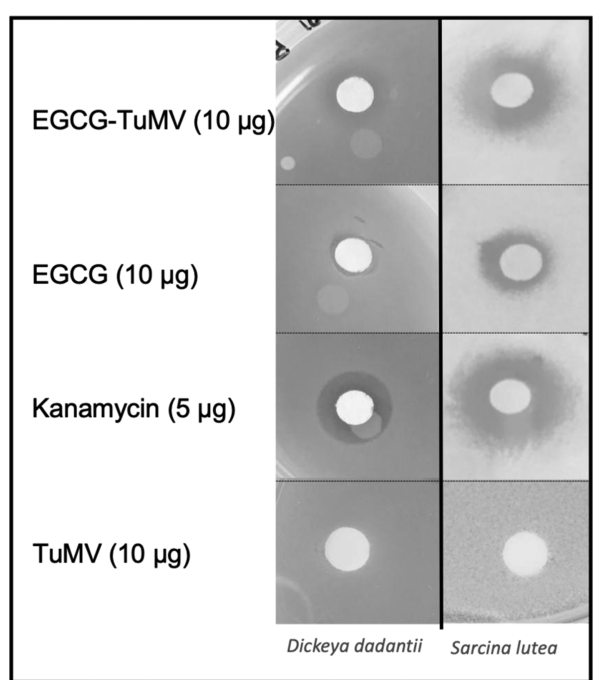

Figure 4. Inhibition of bacterial growth by EGCG-TuMV in solid media plates. Inhibition haloes were obtained for both $D$. dadantii and S. lutea. The inoculum was adjusted to an $\mathrm{OD}_{600}$ of 0.1 and cultured overnight on HMA. Kanamycin was used as a positive inhibition control.

for D. dadantii and P. aeruginosa and MH medium for S. lutea. For all strains tested, as shown in Figure 5, the absorbance of overnight cultures showed the same behavior. Culture growth was notably lower in the cases of higher concentration of EGCG-TuMV nanoparticles, suggesting that the antimicrobial effect of the nanoparticles is concentration-dependent. The observed antimicrobial effect of the EGCG-TuMV nanoparticles can be attributed to the conjugation of the polyphenolic molecule, given that cultures exposed to purified TuMV did not show growth inhibition (Figure 5) and those VNPs produced without the presence of formaldehyde showed very low antimicrobial activity (Figure S2b). A difference in the antimicrobial activity of free EGCG vs EGCG-TuMV particles was observed, with free EGCG moderately inhibiting tested cultures.

The minimum inhibitory concentrations (MICs) shown in Figure 6 were established as the minimum concentration at which $99.9 \%$ of bacterial growth was inhibited. First, cultures were performed in a 96-well microtiter plate with minimal M9 or $\mathrm{MH}$ media and incubated overnight at $37{ }^{\circ} \mathrm{C}$. After incubation, a single plate-serial dilution spotting (SP-SDS) was performed. The MICs found for $D$. dadantii, $P$. aeruginosa, and S. lutea were $16.7,50.0$, and $24.8 \mu \mathrm{g} / \mathrm{mL}$, respectively. Direct inoculation from these inhibited cultures did not yield any colonies, indicating that EGCG-TuMV nanoparticles are bactericidal, an observation that correlates with prior studies on the antimicrobial effects of free EGCG. ${ }^{40}$ In view of this, values for MICs and minimum bactericidal concentrations $(\mathrm{MBC})$ were very similar (Figure 6).

The MIC determinations confirmed that the inhibitory effects of EGCG-TuMV particles were higher than those of free EGCG for all bacteria tested. In the case of $P$. aeruginosa, a MIC of $400 \mu \mathrm{g} / \mathrm{mL}$ has been reported for extracted EGCG, ${ }^{39}$ which is still much higher than $50 \mu \mathrm{g} / \mathrm{mL}$ we found for EGCGTuMV. 

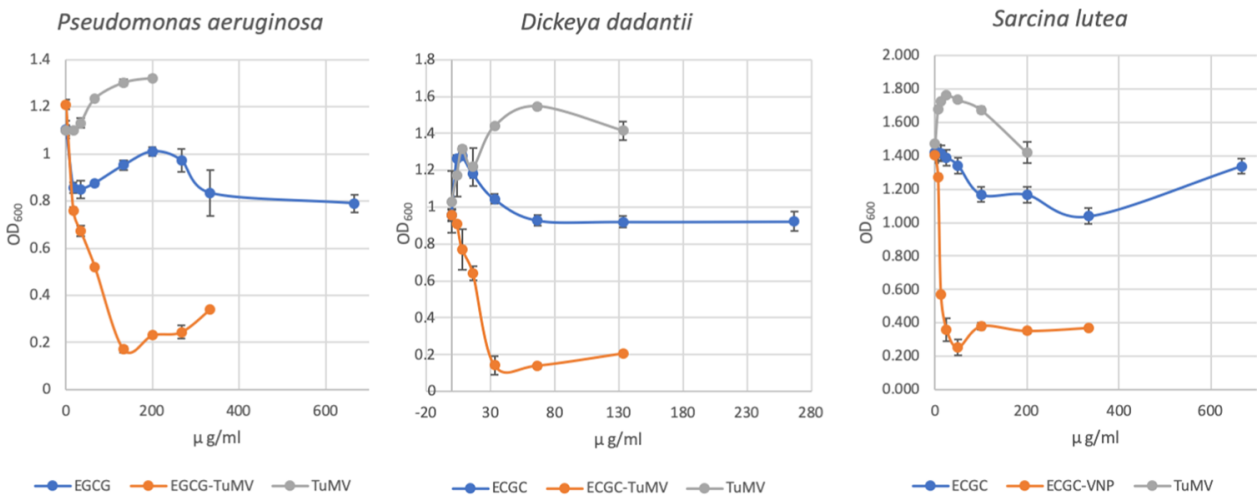

Figure 5. Inhibition of bacterial growth by EGCG-TuMV in liquid cultures. Graphs represent the absorbance (OD $600 \mathrm{~nm})$ of $P$. aeruginosa, D. dadantii, or $S$. lutea cultures after exposure $\mathrm{o} / \mathrm{n}$ to increasing concentrations of free EGCG, TuMV, or EGCG-TuMV.

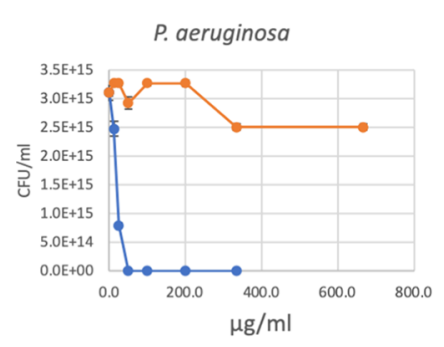

$\rightarrow$ EGCG-TUMV $\rightarrow$ EGCG

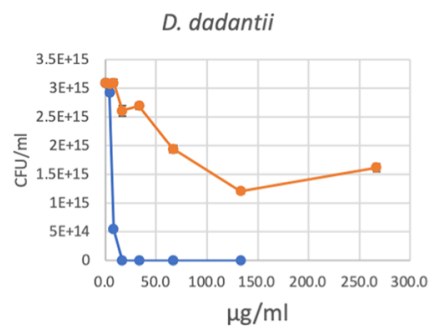

$\rightarrow$ EGCG-TUMV $\rightarrow$ EGCG

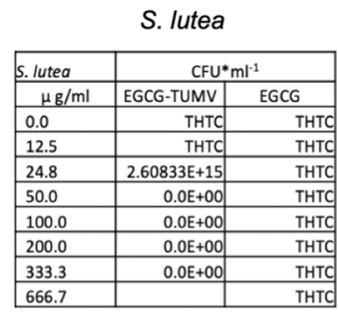

666.7

Figure 6. Quantification of inhibition through colony-forming unit (CFU) counting after bacterial growth in liquid media. CFU count of $P$. aeruginos $a$ and $D$. dadantii after overnight incubation a $37{ }^{\circ} \mathrm{C}$. The results for $S$. lutea are shown in a numerical form because in most cases, the results were too high to count (THTC).

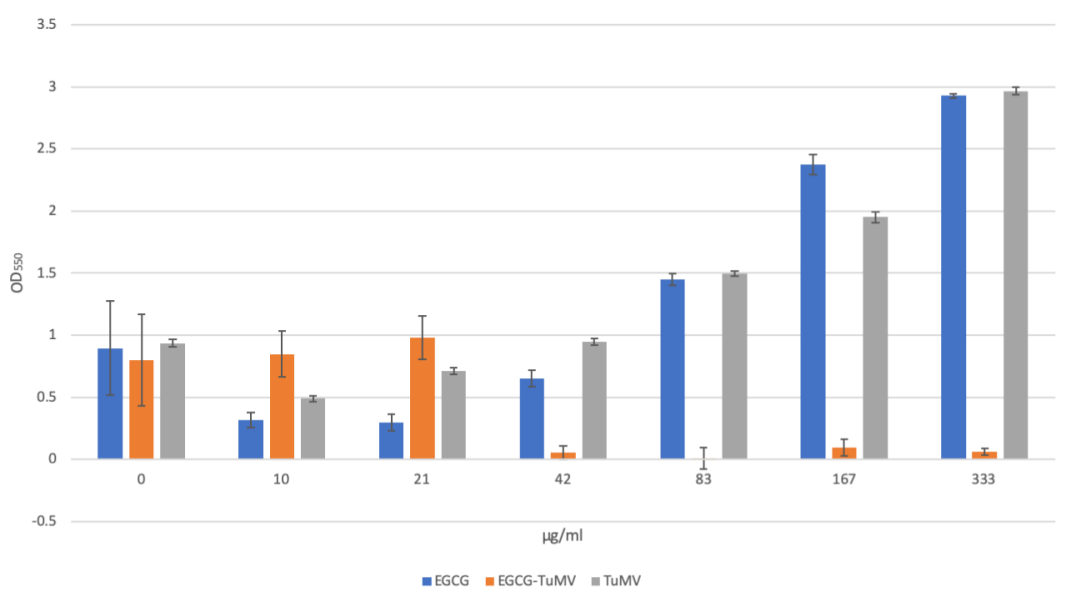

Figure 7. Biofilm adhesion assay of P. aeruginosa. Cultures were grown for $24 \mathrm{~h}$ at $36{ }^{\circ} \mathrm{C}$ in 96 -well microtiter plates containing M9 media. Biofilm formation, indicated by crystal violet staining, was measured at $550 \mathrm{~nm}$.

Contrary to previous reports on a higher susceptibility to EGCG by Gram-positive bacteria, we found that $D$. dadantii (Gram-negative) had a lower MIC in comparison to S. lutea (Gram-positive). Given that similar results have also been found in other studies, ${ }^{41}$ it appears that generalizations regarding differential sensitivity to EGCG by Gram-positive and Gram-negative bacteria are still premature.

Within the concentration range tested, free EGCG did not show complete inhibition of culture growth for any of the bacteria tested. Since the same batch of purified EGCG was used both for these tests and for nanoparticle synthesis, other factors that affect free EGCG stability, such as $\mathrm{pH}$, exposure to light, and susceptibility to redox reactions could play a role. It has been reported that coupling EGCG to protein stabilizes the polyphenolic compound under physiologic conditions. ${ }^{25}$ The latter, together with the exposed nature of EGCG molecules conjugated on the surface of the particle, might explain the difference in antimicrobial effectiveness observed in the present study. Finally, as seen in Figure S4, EGCG-VNP retained good antimicrobial activity after six months of being produced and stored at $4{ }^{\circ} \mathrm{C}$ in $10 \mathrm{mM}$ HEPES.

Evaluation of Biofilm Formation. Bacterial biofilms are multicellular aggregates in which cells are embedded in an extracellular matrix, much more refractory to antimicrobials. This matrix encases bacterial cells and provides protection by performing different tasks. It confers structural stability and 
flexibility, traps antimicrobial compounds, and maintains homeostatic conditions within the biofilm. ${ }^{4}$ Because of this biofilm-conferred infection resilience, the need of new antimicrobial agents addressing this problem has become evident. Biofilm-associated antimicrobial resistance is not acquired by classical antibiotic resistance such as mutations in antibiotic targets but is part of normal bacterial regulatory repertoire. $^{42}$ Specifically, EGCG is an attractive candidate since EGCG-induced bacterial membrane damage deters the ability of the bacteria to bind to each other to form biofilms. ${ }^{43}$

EGCG-TuMV's ability to inhibit biofilm formation was tested using cultures of $P$. aeruginosa in M9 supplemented with glucose exposed to different concentrations of EGCG and EGCG-TuMV and incubated for $24 \mathrm{~h}$, after which the biofilm was quantitated with crystal violet. As shown in Figure 7, EGCG-TuMV VNPs were capable to inhibit the $P$. aeruginosa biofilm formation at a concentration of $42 \mu \mathrm{g} / \mathrm{mL}$. The biofilm inhibition was dependent on EGCG-TuMV VNP concentration and clearly different from the effects found with free EGCG and TuMV treatments.

EGCG has been reported to act against $P$. aeruginosa biofilms by interfering with the assembly of amyloid fibers and impairing quorum sensing. ${ }^{44,45}$ These effects produce biofilms that are less stiff and more susceptible to antibiotics. On the other hand, it has also been reported that EGCG interferes with $P$. aeruginosa swarming motility and enhances the biofilm formation. ${ }^{46}$ The latter was also found in our tests. Free EGCG enhanced the biofilm formation in a concentration-dependent manner (Figure 7). However, nanoparticles loaded with EGCG clearly inhibited the formation of the biofilm of $P$. aeruginosa.

Perspectives. The conjugation of EGCG to TuMV does not seem to impair its capacity to act as an antimicrobial. Rather, it promotes it. Several mechanisms of action have been reported for EGCG, and the compound affects bacteria in different ways. It inhibits enzymes such as dihydrofolate reductase and DNA gyrase, which affect DNA. ${ }^{36}$ It also causes oxidative stress by generating $\mathrm{H}_{2} \mathrm{O}_{2}{ }^{47}$ and binds to both proteins and phospholipids from the lipid bilayer, damaging the cell membrane and increasing the permeability. ${ }^{48}$ Since EGCG has a high affinity for cell membranes and different mechanisms of action have been reported at different levels in the cell, it appears that there is still a lot to be learned about the complete scope of its effects. Although we were able to demonstrate that EGCG-TuMV nanoparticles have a potential as an antimicrobial agent, the actual mechanisms of their inhibitory action are yet to be characterized. Because of the length of TuMV nanoparticles, ca. $700 \mathrm{~nm}$, EGCG-TuMV nanoparticles are more likely to interact outside the cell membrane. Further studies are required to understand whether fragments or complete EGCG-TuMV particles are capable to pass through the cell membrane and to have an effect from within. Nevertheless, for both antimicrobial and antibiofilm properties, EGCG-TuMV VNPs did not only maintain the EGCG activity but also a showed a notable enhancement.

Antimicrobial resistance is a rising problem that needs to be addressed promptly. One of the major advantages of VNPs is the versatility they provide. This technological platform may allow a rapid response to the increasing list of drug-resistant microbes. VNPs not only have a number of sites that can be modified but also there is an array of preparation methods for genetic and chemical manipulations with a high flexibility of applications. ${ }^{49}$ VNPs could be used to address this problem in a number of ways. For instance, an additional target molecule could be conjugated to the nanoparticle, which may increase the amount of antibiotic in an infected site. Likewise, the design of the VNPs with an additional antimicrobial may boost their antimicrobial effect. Specifically, TuMV has proven to have a potential for multiple functionalization. ${ }^{35}$ This versatility, in combination with the results obtained in this study, makes TuMV VNPs good candidates to explore different arrays of molecules to act synergistically with EGCG.

\section{CONCLUSIONS}

The present study showed that EGCG-TuMV nanoparticles produced via Mannich condensation maintain the TuMV structure after the addition of the polyphenolic compound. The EGCG-TuMV nanoparticles proved to be functional, with excellent antimicrobial and antibiofilm properties. Higher antimicrobial susceptibility of Gram-positive bacteria over Gram-negative bacteria was not found. While free EGCG promoted biofilm formation within the range of concentrations tested, EGCG-TuMV showed the opposite effect of inhibiting its formation. In all bacteria tested, susceptibility to EGCGTuMV was higher than to free EGCG. EGCG-TuMV VNPs have the potential to act as antimicrobial agents that enhance EGCG's antimicrobial activities.

\section{ASSOCIATED CONTENT}

\section{Supporting Information}

The Supporting Information is available free of charge at https://pubs.acs.org/doi/10.1021/acsabm.9b01161.

Structural TuMV model (Figure S1); EGCG-VNP characterization in function of the presence of formaldehyde (Figure S2); inhibition after overnight liquid culture (Figure S3); and EGCG-VNP antimicrobial activity at different times after conjugation (Figure S4) (PDF)

\section{AUTHOR INFORMATION}

\section{Corresponding Author}

Fernando Ponz - Centro de Biotecnología y Genómica de Plantas, Universidad Politénica de Madrid-Instituto Nacional de Investigación y Tecnología Agraria y Alimentaria (CBGP, UPM-INIA), 28223 Madrid, Spain; 10 orcid.org/0000-00030344-4363; Email: fponz@inia.es

\section{Authors}

Edith Velázquez-Lam - Centro de Biotecnología y Genómica de Plantas, Universidad Politécnica de Madrid-Instituto Nacional de Investigación y Tecnologia Agraria y Alimentaria (CBGP, UPM-INIA), 28223 Madrid, Spain; Doctorado en Biotecnologia y Recursos Genéticos de Plantas y Microorganismos Asociados, ETSI Agronómica, Alimentaria y de Biosistemas, Universidad Politécnica de Madrid, 28040 Madrid, Spain

Juan Imperial - Instituto de Ciencias Agrarias, CSIC, 28006 Madrid, Spain

Complete contact information is available at: https://pubs.acs.org/10.1021/acsabm.9b01161

\section{Author Contributions}

F.P. and E.V.-L. conceived the presented idea. All assays were performed by E.V.-L., who also proposed the first drafts of the manuscript and contributed to its final form. J.I. suggested the 
microbiological approaches to the assays and the bacteria to be used in them. He supervised the final manuscript versions. F.P. supervised the experiments and assays. He suggested the manuscript structure and supervised its final form.

\section{Notes}

The authors declare no competing financial interest.

\section{ACKNOWLEDGMENTS}

This research was partially funded by RTA2015-00017 (INIA) and APCIN2016-00014-00-00 (Nanobioagri, ArimNet 2) to F.P. The authors thank the Spanish Ministry of Science for the Severo Ochoa Excellence Accreditations to the CBGP (SEV2016-0672). E.V.-L. would like to thank CONACYT and Nuevo Leon Institute for Innovation and Technology Transference for the $\mathrm{PhD}$, student grant (No. 459134, CVU 360539). We thank Lucia Zurita for technical assistance, Carmen Yuste for providing TuMV protein representations, and Flora Sánchez for her supervision. We also thank José Palacios and Emilia López Solanilla for providing S. lutea and D. Dadantii, respectively.

\section{ABBREVIATIONS}

EGCG, (-)-epigallocatechin gallate; VNPs, viruslike nanoparticles; TuMV, Turnip mosaic virus; NP, nanoparticle

\section{REFERENCES}

(1) Coates, A.; Hu, Y.; Bax, R.; Page, C. The Future Challenges Facing the Development of New Antimicrobial Drugs. Nat. Rev. Drug Discovery 2002, 1, 895-910.

(2) Pelgrift, R. Y.; Friedman, A. J. Nanotechnology as a Therapeutic Tool to Combat Microbial Resistance. Adv. Drug Delivery Rev. 2013, 65, 1803-1815.

(3) Aslam, B.; Wang, W.; Arshad, M. I.; Khurshid, M.; Muzammil, S.; Rasool, M. H.; Nisar, M. A.; Alvi, R. F.; Aslam, M. A.; Qamar, M. U.; Salamat, M. K. F.; Baloch, Z. Antibiotic Resistance: A Rundown of a Global Crisis. Infect. Drug Resist. 2018, 11, 1645-1658.

(4) Flemming, H. C.; Wingender, J.; Szewzyk, U.; Steinberg, P.; Rice, S. A.; Kjelleberg, S. Biofilms: An Emergent Form of Bacterial Life. Nat. Rev. Microbiol. 2016, 14, 563-575.

(5) Lamichhane, J. R.; Arendse, W.; Dachbrodt-Saaydeh, S.; Kudsk, P.; Roman, J. C.; van Bijsterveldt-Gels, J. E. M.; Wick, M.; Messéan, A. Challenges and Opportunities for Integrated Pest Management in Europe: A Telling Example of Minor Uses. Crop Prot. 2015, 74, 4247.

(6) Keller, A. A.; Adeleye, A. S.; Conway, J. R.; Garner, K. L.; Zhao, L.; Cherr, G. N.; Hong, J.; Gardea-Torresdey, J. L.; Godwin, H. A.; Hanna, S.; Ji, Z.; Kaweeteerawat, C.; Lin, S.; Lenihan, H. S.; Miller, R. J.; Nel, A. E.; Peralta-Videa, J. R.; Walker, S. L.; Taylor, A. A.; TorresDuarte, C.; Zink, J. I.; Zuverza-Mena, N. Comparative Environmental Fate and Toxicity of Copper Nanomaterials. NanoImpact 2017, 7, $28-40$.

(7) Hui, X.; Hua, S. H.; Wu, Q. Q.; Li, H.; Gao, W. Y. Antimicrobial Mechanism of Epigallocatechin Gallate and Gallocatechin Gallate: They Target 1-Deoxy-D-Xylulose 5-Phosphate Reductoisomerase, the Key Enzyme of the MEP Terpenoid Biosynthetic Pathway. Arch. Biochem. Biophys. 2017, 622, 1-8.

(8) Taylor, P. W.; Hamilton-Miller, J. M. T.; Stapleton, P. D. Antimicrobial Properties of Green Tea Catechins. Food Sci. Technol. Bull.: Funct. Foods 2005, 2, 71-81.

(9) Gopal, J.; Muthu, M.; Paul, D.; Kim, D.-H.; Chun, S. Bactericidal Activity of Green Tea Extracts: The Importance of Catechin Containing Nano Particles. Sci. Rep. 2016, 6, No. 19710.

(10) Zeng, L.; Ma, M.; Li, C.; Luo, L. Stability of Tea Polyphenols Solution with Different $\mathrm{PH}$ at Different Temperatures. Int. J. Food Prop. 2017, 20, 1-18.
(11) Nadim, M.; Auriol, D.; Lamerant-FayeL, N.; Lefe, F.; Dubanet, L.; Redziniak, G.; Kieda, C.; Grillon, C. Improvement of Polyphenol Properties upon Glucosylation in a UV-Induced Skin Cell Ageing Model. Int. J. Cosmet. Sci. 2014, 36, 579-587.

(12) Chandra, G. K.; Tripathy, D. R.; Dasgupta, S.; Roy, A. Interaction of (-)-Epigallocatechin Gallate with Lysozyme-Conjugated Silver Nanoparticles. Appl. Spectrosc. 2012, 66, 744-749.

(13) Sutradhar, P.; Saha, M.; Maiti, D. Microwave Synthesis of Copper Oxide Nanoparticles Using Tea Leaf and Coffee Powder Extracts and Its Antibacterial Activity. J. Nanostruct. Chem. 2014, 4, No. 86.

(14) Chavva, S. R.; Deshmukh, S. K.; Kanchanapally, R.; Tyagi, N.; Coym, J. W.; Singh, A. P.; Singh, S. Epigallocatechin Gallate-Gold Nanoparticles Exhibit Superior Antitumor Activity Compared to Conventional Gold Nanoparticles: Potential Synergistic Interactions. Nanomaterials 2019, 9, 1-16.

(15) Suresh, D.; Udayabhanu; Nethravathi, P. C.; Lingaraju, K.; Rajanaika, H.; Sharma, S. C.; Nagabhushana, H. EGCG Assisted Green Synthesis of $\mathrm{ZnO}$ Nanopowders: Photodegradative, Antimicrobial and Antioxidant Activities. Spectrochim. Acta, Part A 2015, 136, 1467-1474.

(16) Tighe-Neira, R.; Carmora, E.; Recio, G.; Nunes-Nesi, A.; Reyes-Diaz, M.; Alberdi, M.; Rengel, Z.; Inostroza-Blancheteau, C. Metallic Nanoparticles Influence the Structure and Function of the Photosynthetic Apparatus in Plants. Plant Physiol. Biochem. 2018, 130, 408-417.

(17) Kruk, T.; Szczepanowicz, K.; Stefańska, J.; Socha, R. P.; Warszyński, P. Synthesis and Antimicrobial Activity of Monodisperse Copper Nanoparticles. Colloids Surf., B 2015, 128, 17-22.

(18) Hulkoti, N. I.; Taranath, T. C. Biosynthesis of Nanoparticles Using Microbes-A Review. Colloids Surf., B 2014, 121, 474-483.

(19) Zazo, H.; Millán, C. G.; Colino, C. I.; Lanao, J. M. Applications of Metallic Nanoparticles in Antimicrobial Therapy; Elsevier Inc., 2017.

(20) Smith, A.; Giunta, B.; Bickford, P. C.; Fountain, M.; Tan, J.; Shytle, R. D. Nanolipidic Particles Improve the Bioavailability and $\alpha$ Secretase Inducing Ability of Epigallocatechin-3-Gallate (EGCG) for the Treatment of Alzheimer's Disease. Int. J. Pharm. 2010, 389, 207212.

(21) Shtay, R.; Keppler, J. K.; Schrader, K.; Schwarz, K. Encapsulation of (-)-Epigallocatechin-3-Gallate (EGCG) in Solid Lipid Nanoparticles for Food Applications. J. Food Eng. 2019, 244, 91-100.

(22) Silva, A. M.; Martins-Gomes, C.; Fangueiro, J. F.; Andreani, T.; Souto, E. B. Comparison of Antiproliferative Effect of Epigallocatechin Gallate When Loaded into Cationic Solid Lipid Nanoparticles against Different Cell Lines. Pharm. Dev. Technol. 2019, 24, 12431249.

(23) Lei, F.; Wang, X.; Liang, C.; Yuan, F.; Gao, Y. Preparation and Functional Evaluation of Chitosan-EGCG Conjugates. J. Appl. Polym. Sci. 2014, 131, 1-8.

(24) Chen, X.; Du, Y.; Wu, L.; Xie, J.; Chen, X.; Hu, B.; Wu, Z.; Yao, Q.; Li, Q. Effects of Tea-Polysaccharide Conjugates and Metal Ions on Precipitate Formation by Epigallocatechin Gallate and Caffeine, the Key Components of Green Tea Infusion. J. Agric. Food Chem. 2019, 67, 3744-3751.

(25) Li, Z.; Ha, J.; Zou, T.; Gu, L. Fabrication of Coated Bovine Serum Albumin (BSA)-Epigallocatechin Gallate (EGCG) Nanoparticles and Their Transport across Monolayers of Human Intestinal Epithelial Caco-2 Cells. Food Funct. 2014, 5, 1278-1285.

(26) Su, J.; Guo, Q.; Chen, Y.; Mao, L.; Gao, Y.; Yuan, F. Utilization of $\beta$-Lactoglobulin- (-)-Epigallocatechin- 3-Gallate(EGCG) Composite Colloidal Nanoparticles as Stabilizers for Lutein Pickering Emulsion. Food Hydrocoll. 2020, 98, No. 105293.

(27) Malekhosseini, P.; Alami, M.; Khomeiri, M.; Esteghlal, S.; Nekoei, A. R.; Hosseini, S. M. H. Development of Casein-Based Nanoencapsulation Systems for Delivery of Epigallocatechin Gallate and Folic Acid. Food Sci. Nutr. 2019, 7, 519-527.

(28) Xue, J.; Tan, C.; Zhang, X.; Feng, B.; Xia, S. Fabrication of Epigallocatechin-3-Gallate Nanocarrier Based on Glycosylated Ca- 
sein: Stability and Interaction Mechanism. J. Agric. Food Chem. 2014, 62, 4677-4684.

(29) Verma, D.; Gulati, N.; Kaul, S.; Mukherjee, S.; Nagaich, U. Protein Based Nanostructures for Drug Delivery. J. Pharm. 2018, 2018, 1-18.

(30) Cuesta, R.; Yuste-Calvo, C.; Gil-Cartón, D.; Sánchez, F.; Ponz, F.; Valle, M. Structure of Turnip Mosaic Virus and Its Viral-like Particles. Sci. Rep. 2019, 9, No. 15396.

(31) Sánchez, F.; Ponz, F. Presenting Peptides at the Surface of Potyviruses in Planta. In Virus-Derived Nanoparticles for Advanced Technologies. Methods in Molecular Biology; Wege, C.; Lomonossoff, G., Eds.; Humana Press: New York, NY, 2018; Vol. 1776, pp 471485.

(32) Makkar, H. Quantification of Tannins in Tree Foliage; Joint FAO/LAEA Division of Nuclear Techniques in Food and Agriculture: Vienna, Austria, 2000.

(33) Makkar, H.; Dawra, R. K.; Singh, B. Determination of Both Tannin and Protein in a Tannin-Protein Complex. J. Agric. Food Chem. 1988, 36, 523-525.

(34) Thomas, P.; Sekhar, A. C.; Upreti, R.; Mujawar, M. M.; Pasha, S. S. Optimization of Single Plate-Serial Dilution Spotting (SP-SDS) with Sample Anchoring as an Assured Method for Bacterial and Yeast Cfu Enumeration and Single Colony Isolation from Diverse Samples. Biotechnol. Rep. 2015, 8, 45-55.

(35) Yuste-Calvo, C.; González-Gamboa, I.; Pacios, L. F.; Sánchez, F.; Ponz, F. Structure-Based Multifunctionalization of Flexuous Elongated Viral Nanoparticles. ACS Omega 2019, 4, 5019-5028.

(36) Das, S.; Tanwar, J.; Hameed, S.; Fatima, Z. Antimicrobial Potential of Epigallocatechin-3- Gallate (EGCG): A Green Tea Polyphenol. J. Biochem. Pharmacol. Res. 2014, 2, 167-174.

(37) Wang, X.; Zhang, J.; Lei, F.; Liang, C.; Yuan, F.; Gao, Y. Covalent Complexation and Functional Evaluation of (-)-Epigallocatechin Gallate and $\alpha$-Lactalbumin. Food Chem. 2014, 150, 341-347.

(38) Hagerman, A. E. Fifty Years of Polyphenol-Protein Complexes. In Recent Advances in Polyphenol Research; Cheynier, V.; SarniManchado, P.; Quideau, S., Eds.; Wiley-Blackwell: Oxford, UK, 2012; Vol. 3, pp 71-97.

(39) Jeon, J.; Kim, J. H.; Lee, C. K.; Oh, C. H.; Song, H. J. The Antimicrobial Activity of (-)-Epigallocatehin-3-Gallate and Green Tea Extracts against Pseudomonas aeruginosa and Escherichia coli Isolated from Skin Wounds. Ann. Dermatol. 2014, 26, 564-569.

(40) Ikigai, H.; Nakae, T.; Hara, Y.; Shimamura, T. Bactericidal Catechins Damage the Lipid Bilayer. Biochim. Biophys. Acta 1993, $1147,132-136$.

(41) Sun, L. M.; Zhang, C. L.; Li, P. Characterization, Antimicrobial Activity, and Mechanism of a High-Performance (-)-Epigallocatechin3-Gallate (EGCG)-CuII/Polyvinyl Alcohol (PVA) Nanofibrous Membrane. J. Agric. Food Chem. 2011, 59, 5087-5092.

(42) Hengge, R. Targeting Bacterial Biofilms by the Green Tea Polyphenol EGCG. Molecules 2019, 24, No. 2403.

(43) Blanco, A. R.; Sudano-Roccaro, A.; Spoto, G. C.; Nostro, A.; Rusciano, D. Epigallocatechin Gallate Inhibits Biofilm Formation by Ocular Staphylococcal Isolates. Antimicrob. Agents Chemother. 2005, 49, 4339-4343.

(44) Stenvang, M.; Dueholm, M. S.; Vad, B. S.; Seviour, T.; Zeng, G.; Geifman-Shochat, S.; Søndergaard, M. T.; Christiansen, G.; Meyer, R. L.; Kjelleberg, S.; Nielsen, P. H.; Otzen, D. E. Epigallocatechin Gallate Remodels Overexpressed Functional Amyloids in Pseudomonas aeruginosa and Increases Biofilm Susceptibility to Antibiotic Treatment. J. Biol. Chem. 2016, 291, 26540-26553.

(45) Yang, L.; Liu, Y.; Sternberg, C.; Molin, S. Evaluation of EnoylAcyl Carrier Protein Reductase Inhibitors as Pseudomonas Aeruginosa Quorum-Quenching Reagents. Molecules 2010, 15, No. 780 .

(46) O’May, C.; Ciobanu, A.; Lam, H.; Tufenkji, N. Tannin Derived Materials Can Block Swarming Motility and Enhance Biofilm Formation in Pseudomonas aeruginosa. Biofouling 2012, 28, 10631076.
(47) Cui, Y.; Oh, Y. J.; Lim, J.; Youn, M.; Lee, I.; Pak, H. K.; Park, W.; Jo, W.; Park, S. AFM Study of the Differential Inhibitory Effects of the Green Tea Polyphenol (-)-Epigallocatechin-3-Gallate (EGCG) against Gram-Positive and Gram-Negative Bacteria. Food Microbiol. 2012, 29, 80-87.

(48) Papuc, C.; Goran, G. V.; Predescu, C. N.; Nicorescu, V.; Stefan, G. Plant Polyphenols as Antioxidant and Antibacterial Agents for Shelf-Life Extension of Meat and Meat Products: Classification, Structures, Sources, and Action Mechanisms. Compr. Rev. Food Sci. Food Saf. 2017, 16, 1243-1268.

(49) Alemzadeh, E.; Dehshahri, A.; Izadpanah, K.; Ahmadi, F. Plant Virus Nanoparticles: Novel and Robust Nanocarriers for Drug Delivery and Imaging. Colloids Surf., B 2018, 167, 20-27. 